\title{
Ethischer Konflikt im Basler Frauenspital. Diakonissen nahmen Stellung zu Schwangerschafts- abbrüchen
}

Sabine Braunschweig

\section{Summary}

In the 1940s there occurred an ethical conflict in the Women's Hospital of Basle. It arose in the context of a shortage of nurses, the introduction of the Swiss national criminal law, the change of the hospital director, the increase of abortion and the nursing ideal of obedience and serving. The conflict showed the social change towards measures of birth control such as abortion and sterilisation. Different political opinions and strong convictions clashed. The paper is focusing on denominationally affiliated nurses, the deaconesses of Riehen, who were standing between the religious conviction to protect unborn life and the professional principle of unconditional nursing. Finally they decided to leave the hospital.

Keywords: ethics of care; history of nurses; Women's Hospital of Basle; abortion; sterilisation

\section{Zusammenfassung}

Im Spannungsfeld von Pflegepersonalmangel, Einführung des eidgenössischen Strafgesetzbuches, Wechsel in der Spitaldirektion, Zunahme der Schwangerschaftsabbrüche sowie dem Schwesternideal von Gehorsam und Dienen spielte sich im Basler Frauenspital Mitte der 1940er Jahre ein ethischer Konflikt ab, bei dem ein gesellschaftlicher Wandlungsprozess um Massnahmen der Geburtenregelung wie Abtreibung und Sterilisation sichtbar wurde. Bei der Frage der Geburtenkontrolle prallten verschiedene Auf-

Sabine Braunschweig, Büro für Sozialgeschichte, Dornacherstrasse 192, CH-4053 Basel (sbraunschweig@vtxmail.ch). 
fassungen aufeinander. Im Fokus stehen konfessionell gebundene Krankenschwestern des Diakonissenhauses Riehen, die ihrer religiösen Überzeugung in bezug auf den Schutz des ungeborenen Lebens vor dem beruflichen Grundsatz der bedingungslosen Pflege den Vorrang gaben und schliesslich das Frauenspital verliessen.

Im Spannungsfeld von Pflegepersonalmangel, Einführung des eidgenössischen Strafgesetzbuches, Wechsel in der Spitaldirektion, Zunahme der Schwangerschaftsabbrüche sowie dem Schwesternideal von Gehorsam und Dienen spielte sich im Basler Frauenspital Mitte der 1940er Jahre ein ethischer Konflikt ab, den die Beteiligten und Betroffenen auf je unterschiedliche Weise angingen. Es ging dabei um Massnahmen der Geburtenregelung wie Abtreibung und Sterilisation. Diese Operationen wurden im Operationssaal der gynäkologischen Abteilung des Frauenspitals vorgenommen. Sowohl für einen Teil der Ärzte und Krankenschwestern wie auch für viele schwangere Frauen waren diese Eingriffe mit schwerwiegenden moralischethischen Fragen, Zweifeln und Ängsten verbunden.

Als pflegegeschichtliche Arbeit will dieser Beitrag untersuchen, welchen widersprüchlichen Idealen und Anforderungen die Krankenschwestern in ihrer Tätigkeit genügen mussten, welcher Handlungsspielraum ihnen zur Verfügung stand und wie sie ihn ausnutzten, welche Unterstützung sie erhielten und zu welchem Entscheid dies führte.

Es werden in diesem Konflikt gesellschaftliche Wandlungsprozesse sichtbar, die zu stärkerer Entscheidungskompetenz führten, die aber auch auf gesellschaftlichen Widerstand stiessen. Als Quellen wurden die Akten des Frauenspitals Basel und des Diakonissenhauses Riehen sowie zeitgenössische Pflegezeitschriften ausgewertet ${ }^{1}$.

\section{Pflege durch Diakonissen}

Wie in der ersten Hälfte des 20. Jahrhunderts in vielen Krankenhäusern noch üblich arbeiteten auch im Basler Frauenspital konfessionell gebundene Schwestern. Seit 1873 waren es Riehener Diakonissen, die zunächst in

1 Dieser Beitrag entstand im Rahmen des NF-Projekts «Eugenik in Psychiatrie und Verwaltung in Basel-Stadt, 1880-1960» unter Leitung von Prof. Regina Wecker im Nationalen Forschungsprogramm 51 «Integration und Ausschluss». Ich danke meinen Kolleginnen und Kollegen für ihre wertvollen Anregungen. 
der Wöchnerinnenabteilung des Basler Bürgerspitals den Pflegedienst verrichteten. Von der deutschen Kaiserswerther Diakonie ${ }^{2}$ ausgehend wurde in Riehen bei Basel 1852 eine weitere Diakonissenanstalt in der Schweiz gegründet. Die heute noch bestehende reformierte Schwesterngemeinschaft verpflichtet sich zu Gehorsam in Mündigkeit, Ehelosigkeit und Anspruchslosigkeit und lebt nach der aus dem 6. Jahrhundert stammenden Benediktregel $^{3}$. Zunächst widmete sich die Lebens-, Glaubens- und Dienstgemeinschaft der Krankenpflege und gründete in Riehen ein eigenes Spital, das heutige Gemeindespital, später übernahm sie weitere soziale und pflegerische Aufgaben an verschiedenen Orten ${ }^{4}$. Wie katholische Ordensgemeinschaften hatten auch die reformierten Diakonissenhäuser schriftliche Vereinbarungen, sogenannte Stationsverträge, mit den Institutionen, in denen sie tätig waren.

Als 1896 das Frauenspital in Basel eröffnet wurde, schloss die Direktion mit dem Komitee des Diakonissenhauses Riehen einen Vertrag ab, worin sich dieses verpflichtete, dem Frauenspital Diakonissen als Hebammen und Krankenschwestern zur Verfügung zu stellen ${ }^{5}$. Die Stellen der Ober- und Unterhebamme der Wöchnerinnenabteilung sowie der Oberschwester der gynäkologischen Abteilung, also die leitenden Posten, sollten im gegenseitigen Einverständnis zwischen Frauenspital und Diakonissenhaus besetzt werden (§ 2). Die Spitalleitung wünschte sich «tüchtige Schwestern, worunter auch noch nicht vollständig geübte, jedoch bildungsfähige verstanden» (§ 1) wurden. Zur Unterstützung bei den Hausgeschäften wie «Putzen, Fegen, Blochen und derartige schwere Arbeiten ausserhalb der Krankensäle» (§ 7) wurden ihnen Mägde zur Seite gestellt. Explizit wurde auch betont, dass zur Bedienung der Ärzte keine Schwestern verwendet würden (§ 7). Dies musste hervorgehoben werden, weil es in vielen Spitälern zum Pflichtenheft der Pflegenden gehörte, den Ärzten «bei Tisch aufzutragen ${ }^{6}$.

Nichtsdestotrotz unterstanden die Diakonissen, auch wenn sie mit dem Gehorsamsgelübde ihrem Mutterhaus verpflichtet waren, als Krankenschwestern den Ärzten und hatten ärztliche Anordnungen gehorsam und

2 Das Pastorenehepaar Theodor und Friederike Fliedner gründete in Kaiserswerth bei Düsseldorf 1836 analog zu den katholischen Ordensgemeinschaften die erste Diakonissenanstalt, um junge Töchter für die christliche Krankenpflege und Diakonie auszubilden. Vgl. Sticker 1960, $189 \mathrm{ff}$.

3 Die Benediktregel wurde von Benedikt von Nursia (480-546) geschrieben, der als der Begründer des christlichen Mönchtums im Westen gilt.

4 Vgl. Kellerhals/Seiler/Stuber 2002.

5 Staatsarchiv Basel-Stadt (StABS), SD-REG 1a 0-1-3, Frauenspital, Aufsichtskommission, «Vertrag zwischen dem Frauenspital Basel-Stadt einerseits u. der Diakonissenanstalt in Riehen anderseits, 5.2.1896».

6 Vgl. Freudweiler/Oser 1913, 5. 
willig auszuführen, auch wenn die devote Geste der Bedienung bei den Mahlzeiten ausgeklammert war ${ }^{7}$.

\section{Pflegepersonalmangel}

Als in Basel 1942 der Vertrag zwischen dem Diakonissenhaus Riehen und dem Frauenspital zur Erneuerung anstand, zeigten sich bereits die ersten Anzeichen des Pflegepersonalmangels, der mit kleineren und grösseren Schwankungen bis in die 1970er Jahre anhielt ${ }^{8}$. Alle Spitäler in der Schweiz, aber auch in den benachbarten Ländern litten unter personellen Engpässen.

Der Schwesternmangel hatte mehrere Ursachen: Einerseits waren in der ersten Hälfte des 20. Jahrhunderts neue Frauenberufe entstanden, wie etwa der Beruf der Bürolistin oder der Telefonistin, andererseits verlor der Schwesternberuf mit den strengen Arbeitsbedingungen und der niedrigen Entlöhnung an Attraktivität für junge, mittelständische und gut ausgebildete Frauen. Die Verpflichtung zum Zölibat und das Internat wirkten je länger, je mehr abschreckend. Der Pflegeberuf wurde nicht mehr als der ideale Frauenberuf wahrgenommen. Weitere Gründe für den Personalmangel lagen in der Zunahme von Spitalneubauten, die in jenen Jahren zu einem Mehrbedarf an Pflegepersonal führten, sowie im Normalarbeitsvertrag, der 1947 erstmals für das Pflegepersonal in Kraft trat und eine wöchentliche Höchstarbeitszeit von 60 respektive 66 Stunden festlegte, was einer Arbeitszeitverkürzung von zehn bis zwanzig Stunden entsprach und entsprechend mehr Personal erforderte.

Mit verschiedenen Massnahmen versuchten Behörden und Spitalleitungen sowie die Pflegeverantwortlichen diesen Notstand abzumildern. Kurz nach dem Zweiten Weltkrieg wurden Krankenschwestern in Österreich und Deutschland rekrutiert, die der höheren Löhne wegen in die Schweiz kamen. In den 1950er Jahren versuchte man, verheiratete ehemalige Krankenschwestern, die bis anhin bei der Eheschliessung aus dem Beruf ausscheiden mussten, zurückzuholen, und führte die Teilzeitanstellung ein. Als weitere Entlastung der diplomierten Krankenschwestern wurde der Beruf der Spitalgehilfin entwickelt, die vorwiegend im hauswirtschaftlichen Teil Aufgaben wie etwa die Essensausteilung und Essenseingabe, die Blumenbesorgung, das

7 Dalonni 1943, «Wenn der Arzt nicht mit dem Gehorsam und der Zuverlässigkeit der Schwester rechnen kann, so wird ihm die Erfüllung seiner Aufgabe verunmöglicht.» S. 111. Dieser Artikel im Diakonissenbote aus Riehen kann als programmatischer Beitrag zum Verhältnis von Medizin und Pflege gelesen werden.

8 Vgl. im folgenden: Braunschweig 1998. 
Teekochen und leichte Putzarbeiten übernehmen sollte. Um 1962 wurde die neue Berufsausbildung der praktischen Krankenpflege geschaffen, die nicht mit einem Diplom, sondern mit einem vom Schweizerischen Roten Kreuz anerkannten Fähigkeitsausweis abschloss. Doch der Pflegepersonalmangel entschärfte sich erst, als Mitte der 1970er Jahre die wirtschaftliche Entwicklung in den Industrieländern einbrach und die Erwerbslosigkeit zunahm. Dann wurden sichere Staatsstellen in Krankenhäusern begehrt, obwohl die Arbeitsbedingungen der Pflegeberufe noch immer nicht mit äquivalenten Berufen vergleichbar waren.

Seit den 1940er Jahren spürten auch die reformierten Diakonissenhäuser und die katholischen Ordensgemeinschaften den gesellschaftlichen Wandel der Frauenrollen und der weiblichen Lebensformen: Die Eintritte junger Frauen in diese religiösen Institutionen begannen zurückzugehen ${ }^{9}$. Eine Auswirkung war, dass diese den Bedarf an vertraglich zugesicherten Krankenschwestern immer weniger erfüllen konnten, wie sich auch im Frauenspital Basel zeigte. Bei der anstehenden Vertragserneuerung 1942, musste der Paragraph 1 so abgeändert werden, dass die Diakonissenanstalt «diejenige Anzahl von Schwestern übergibt, die sie zur Verfügung stellen kann» ${ }^{10}$. Dies weist darauf hin, dass sie nicht mehr in der Lage war, den Gesamtbestand sicherzustellen. Um eine gewisse Einheitlichkeit zu wahren, sollten aber die Mehrheit der Krankenschwestern nach wie vor Diakonissen sein. Da dies nicht mehr auf allen Abteilungen gewährleistet werden konnte, wurden auf den 1.Juli 1945 die Diakonissen von der «septischen gynäkologischen Abteilung II» abgezogen und durch freie Berufskrankenschwestern ersetz ${ }^{11}$. Trotz dieser Massnahme war die Personalfrage auch künftig nicht gelöst, wie der Vorsteher, Pfarrer Fritz Hoch, im Jahresbericht 1944/45 ans Komitee schrieb, was ich weiter unten ausführlich darlegen werde.

Im Archiv des Diakonissenhauses Riehen findet sich die Korrespondenz zwischen dem Vorsteher und der Leitung des Frauenspitals, die beredt den «Schwesternmangel» aufzeigt.

So beklagte sich beispielsweise 1945 der Verwalter des Frauenspitals, dass drei Schwestern immer noch krank seien und eine im Militärdienst weile, was sich sehr unangenehm und hindernd auf den Dienstbetrieb auswirke. Er

9 Im Diakonissenboten aus Riehen erschienen in den 1940er Jahren regelmässig Klagen über den Rückgang der Neueintritte und Einsegnungen in allen Diakonissenhäusern.

10 Diakonissenhaus Riehen, Archiv, Frauenspital [im folgenden: Archiv], «Vertrag zwischen dem Frauenspital Basel-Stadt und der Diakonissenanstalt in Riehen betreffend Uebernahme der Krankenpflege durch Riehener Schwestern», unterzeichnet von Dr. E. Zweifel, Sanitätsdirektor, am 15.10.1942 und Pfr. F. Hoch, Vorsteher der Diakonissenanstalt Riehen am 14.4.1943.

11 Archiv, Aus dem Jahresbericht 1944/45 ans Komitee von Pfr. Hoch, 243. 
fürchtete, dass diese Zustände «zur Kenntnis unserer vorgesetzten Behörde gelangen oder sogar den Weg in die breite Oeffentlichkeit nehmen» könnten, und verlangte Ersatz für die erkrankten Schwestern ${ }^{12}$. Der Vorsteher antwortete ihm, dass auch das Diakonissenspital unter solchen krankheitsbedingten Abwesenheiten leide, die zudem häufiger auftreten würden als in früheren Jahren. Diese Ergänzung kann als Hinweis darauf gedeutet werden, dass Krankenschwestern, die wegen des allgemeinen Personalmangels öfters auf Ferien und Freistunden verzichten mussten, sich deshalb überarbeiteten und krankheitsanfälliger waren.

Weiter stellte der Vorsteher klar, dass er schon lange Ersatzschwestern geschickt hätte, wenn diese Personalnot nur das Frauenspital beträfe und nicht überall herrschte.

\section{Abtreibung und Sterilisation}

Auf einer anderen Ebene als dem Personalengpass gab es für die Diakonissen, die auf der gynäkologischen Abteilung und im Operationssaal des Frauenspitals Basel arbeiteten, ein schwerwiegendes Problem, das sich 1942 mit dem neuen Strafgesetzbuch und dem Direktionswechsel von Prof. Alfred Labhardt zu Prof. Theodor Koller verschärfte: Dies betraf die Frage der Schwangerschaftsunterbrechungen.

Der Schwangerschaftsabbruch war ein höchst kontroverses Thema ${ }^{13}$. Intensiv hatte das eidgenössische Parlament im März 1929 im Zusammenhang mit der Schaffung des schweizerischen Strafgesetzbuches (StGB) die Abtreibungsfrage debattiert. Unterschiedliche Weltanschauungen prallten aufeinander: Die kommunistische Partei verlangte die vollständige Freigabe, die katholisch-konservative Partei forderte ein Verbot ohne jede Ausnahme. Die sozialdemokratische Partei sprach sich grundsätzlich für die Strafbarkeit der Abtreibung aus, wollte jedoch die medizinische um die juristische, eugenische und die soziale Indikation erweitern. Die Parlamentsmehrheit akzeptierte schliesslich nur die medizinische Indikation, nach der ein Schwangerschaftsabbruch straflos war, wenn die Gesundheit der schwangeren Frau gefährdet war. Am 4. Juli 1938 stimmten die Schweizer Männer nach einem ausserordentlich langen Gesetzgebungsprozess über das neue Strafgesetzbuch ab. Trotz Ablehnung durch die meisten katholischen Kantone, vor allem wegen des fehlenden Abtreibungsverbots, kam die Vorlage knapp durch und das Strafgesetzbuch wurde am 1. Januar 1942 in Kraft gesetzt.

12 Vgl. StABS, Frauenspital, Brief des Verwalters Etterich an Pfr. Hoch, 2.7.1945.

13 Vgl. im folgenden: Joris/Witzig 1986, $321 \mathrm{ff}$. 
Welche Veränderungen zeichneten sich nach Einführung des Strafgesetzbuches im Basler Frauenspital ab? Legale Abtreibungen wurden meist in Krankenhäusern vorgenommen. In Basel hatte bereits im Rahmen der kantonalen Strafrechtsrevision 1918 eine politische Debatte um den straflosen Schwangerschaftsabbruch stattgefunden ${ }^{14}$. Als eine Fristenlösung eingeführt werden sollte, löste dies heftige Reaktionen aus: Im Namen der Basler Medizinischen Gesellschaft lehnte der Direktor des Frauenspitals Alfred Labhardt diese «Freigabe der Abtreibung» als moralisch und medizinisch unverantwortlich ab und verfasste zuhanden des Parlaments einen entsprechenden Antrag ${ }^{15}$. In zweiter Lesung wurde die Vorlage für einen straffreien Schwangerschaftsabbruch vom Basler Grossen Rat schliesslich abgelehnt. Nur bei einer medizinischen Indikation blieb eine Abtreibung erlaubt.

Aus Angst vor der Ablehnung des StGB hatte man darauf verzichtet, die Sterilisation hier zu regeln. Wie bis anhin blieb sie im Operationsrecht geregelt. Als chirurgischer Eingriff galt sie als Körperverletzung, deren Straftatbestand nur aufgehoben wurde, wenn die Einwilligung vorlag, der Eingriff einen therapeutischen Zweck hatte und sachgemäss ausgeführt wurde. Eine Sterilisation konnte jedoch streng genommen keinen therapeutischen Zweck für sich beanspruchen. Neben der medizinischen Indikation waren in den 1920er Jahren zunehmend auch soziale und eugenische Indikationen anerkannt worden. Wegen der doch heiklen rechtlichen Situation erarbeitete die Basler Medizinische Gesellschaft 1934 Richtlinien, die die Bedingungen festlegten, unter denen eine Sterilisation gemacht werden durfte. Ausser im Kanton Waadt wurde in keinem Kanton ein spezifisches Sterilisationsgesetz verabschiedet ${ }^{16}$.

Mit der Einführung des eidgenössischen Strafgesetzes 1942 änderte sich in Basel nur wenig. Bei einer Schwangerschaft und dem Wunsch, diese zu beenden, musste der Gynäkologe die Schwangerschaft bestätigen und die Frau, wenn keine somatisch-medizinische Begründung vorlag, einem Psychiater zuweisen ${ }^{17}$. Dieser klärte ab, ob der Eingriff im Rahmen einer medizinisch-psychiatrischen Indikation durchgeführt werden konnte, und verfasste ein entsprechendes Gutachten. Das Basler Gesundheitsamt vergab die Kompetenz, Gutachten für den Schwangerschaftsabbruch auszustellen. Die dazu berechtigten Ärzte und Ärztinnen mussten alljährlich die Zahl der Gutachten dem Gesundheitsamt mitteilen. Ebenso musste das Frauenspital die Zahl der Abtreibungen für statistische Zwecke weiterleiten.

14 Vgl. im folgenden: Imboden/Ritter 2007.

15 Labhardt 1919.

16 Vgl. für das Sterilisationsgesetz von 1928: Heller/Jeanmonod/Gasser 2002.

17 Vgl. Imboden/Ritter 2007. 
Eine Wegleitung der Medizinischen Gesellschaft zum StGB bestimmte, dass die schwangere Frau und ihr Partner die Einwilligung in die Abtreibung und die eventuelle Sterilisation schriftlich geben mussten, bei Minderjährigen oder urteilsunfähigen Personen deren gesetzlicher Vertreter. Im reformierten Kanton Basel-Stadt etablierte sich eine grosszügige Praxis der Schwangerschaftsunterbrechung und Sterilisation.

\section{Beratung für schwangere Frauen}

Im selben Jahr 1942, als das neue Strafgesetzbuch in Kraft trat, wurde Alfred Labhardt, Direktor des Frauenspitals, emeritiert. Sein Nachfolger, Theo Koller, hatte während fast 18 Jahren in verschiedenen Funktionen, zuletzt als Oberarzt, an der Zürcher Frauenklinik gearbeitet. Er hatte sich insbesondere um die Schwangerenfürsorge verdient gemacht: 1932 hatte er in Zürich den Verein Mütterhilfe ${ }^{18}$ gegründet und 1939 gemeinsam mit Heinrich Willi, dem Leiter des kantonalzürcherischen Säuglingsheims, den erfolgreichen Ratgeber «Die Gesunderhaltung von Mutter und Kind» herausgegeben, der bis in die 1970er Jahre knapp dreissig Auflagen erfuhr ${ }^{19}$.

Kaum hatte Koller sein neues Amt angetreten, wurde er vom Basler Frauenverein angefragt, ob er an der Jahresversammlung vom 29.1.1943 einen Vortrag über die Schweizer Fürsorge halten würde ${ }^{20}$. Er sagte zu und regte an, die Unterstützung schwangerer Frauen auszubauen - dies auch in der Absicht, illegale Abtreibungen, die grosse gesundheitliche Risiken für die schwangeren Frauen bargen, zu vermindern. Im Anschluss an diesen Anlass telefonierte die Assistenzärztin der psychiatrischen Poliklinik, Dr. S. Müller, die als Gutachterin mit den Nöten von schwangeren Frauen konfrontiert war, der Präsidentin des Frauenvereins und betonte «die dringende Notwendigkeit einer vermehrten Fürsorge für die werdende Mutter» ${ }^{21}$.

Der Basler Frauenverein war bereit, sich mit dieser Frage zu befassen, ein entsprechendes Projekt auszuarbeiten und finanzielle Mittel aufzutreiben. Im Oktober 1944 konnte die unentgeltliche «Beratungsstelle für werdende Mütter» auf der Geschäftsstelle am Heuberg eröffnet werden. Das Präsidium der begleitenden Kommission übernahm Frau Gertrud Koller-von Seutter, Gattin des Frauenspitaldirektors ${ }^{22}$. Im Frühjahr 1945 zeichneten sich jedoch

18 Vgl. Berger/Stäuble 1970, 267.

19 Koller/Willi 1939 (291971).

20 StABS, Basler Frauenverein, PA 882, E 1.2, Vorstandsprotokoll, 3.12.1942.

21 StABS, PA 882, E 1.2, Vorstandsprotokoll, 4.2.1943.

22 StABS, PA 882, E 1.2, Vorstandsprotokoll, 4.10., 1.11., 6.12.1944. 
Konflikte zwischen ihr und dem Basler Frauenverein ab. Es ging laut Vorstandsprotokoll um unterschiedliche Auffassungen über die Arbeit der Beratungsstelle, was bereits im Juni 1945 dazu führte, dass sie dem Frauenspital angegliedert wurde ${ }^{23}$. In den folgenden Jahren stieg die Beratungsfrequenz stark $a^{24}$. Mit materieller und ideeller Unterstützung wurde schwangeren Frauen, in der grossen Mehrheit verheirateten Frauen, unter die Arme gegriffen. Aus der von einer Fürsorgerin betreuten «Beratungsstelle für werdende Mütter» entwickelte sich in den folgenden Jahrzehnten die sozialmedizinische Abteilung der Universitätsfrauenklinik Base ${ }^{25}$.

\section{Ethischer Konflikt}

Obwohl Abtreibungen und Sterilisationen seit längerem im Frauenspital ausgeführt wurden und Diakonissen die Pflege ausübten, verschärfte sich für sie die Situation. Mit der neu gesetzlich notwendigen Meldung der Abortzahlen an das Gesundheitsamt, über die die Vizeoberschwester Diakonisse E. R. Buch führte, fiel ihnen die Zunahme von Abtreibungen auf. Wurden laut Zählung der Schwestern 194364 Eingriffe unternommen, waren es 1944 76 und im ersten Halbjahr 1945 bereits 50 Schwangerschaftsabbrüche, wie sie Pfarrer Fritz Hoch schrieben ${ }^{26}$. Dieser war Vorsteher des Diakonissenhauses und diente in dieser Rolle als Vermittler zwischen Mutterhaus und Spitalleitung. Zudem unterstützte er die Diakonissen als Seelsorger bei persönlichen Anliegen. Die Unruhe unter ihnen teilte er dem Komitee des Diakonissenhauses mit:

Unsere Operationsschwestern klagen über die starke Zunahme der Schwangerschaftsunterbrechungen. [...] Unsere Operationsschwestern und zum Teil auch die pflegenden Schwestern fühlen sich durch diese Eingriffe in ihrem mütterlichen Empfinden verletzt und vor allem in ihrem christlichen Gewissen bedrückt. [...] So erhebt sich die Frage, was mit dieser Abteilung geschehen soll. Eine Radikallösung des Problems würde darin bestehen, unter offenem Protest gegen die überhandnehmenden Schwangerschaftsunterbrechungen den ganzen Posten zu kündigen und freien Schwestern zu überlassen. ${ }^{27}$

23 StABS, PA 882, E 1.2, Vorstandsprotokoll, 2.5.1945 und 6.6.1945.

24 Vgl. StABS, GGG, PA 146 Q 25, Beratungsstelle für werdende Mütter: Jahresberichte 1948-1951.

25 Mall-Haefeli 1970, 415.

26 Archiv: Schwester E. R. an Pfarrer Hoch, 17.6.1945. Vgl. StABS, SD-REG 1, 3-0-38 Sterilisation, Abort (1935-1960): Für die Jahre 1943, 1944, 1945 waren dies 249 Aborte im Frauenspital, was den Zahlen von E. R. entspricht, mit 59 zusätzlichen Aborten für den Zeitraum 1.7.-31.12.1945. Diesen Hinweis verdanke ich Gabriela Imboden.

27 Archiv: Jahresbericht 1944/45 von Pfarrer Hoch ans Komitee, S. 243. 
Statt des totalen Rückzugs aller Diakonissen ins Mutterhaus schlug Pfarrer Hoch dann eine Kompromisslösung vor, nämlich die Schwestern aus dem Operationssaal zurückzuziehen und die verbleibenden Diakonissen auf den Abteilungen der Gynäkologie und Geburtshilfe des Frauenspitals zu konzentrieren. Mitte August 1945 hatte er diesen Sachverhalt mit Prof. Koller besprochen. Dieser war mit der Lösung einverstanden, dass die Diakonisse E. R. den Posten der «Etagenschwester» und die Leitung der «gynäkologischen Abteilung I» übernehme und die freie Krankenschwester B. neu mit der Leitung des Operationssaals betraut würde. Pfarrer Hoch legte also die Bedenken der Diakonissen gegenüber der sich häufenden Anzahl Schwangerschaftsabbrüche auf den Tisch und setzte sich bei der Direktion des Frauenspitals für sie ein.

Diakonisse C. C. hatte ihm wenige Tage zuvor auf seine Nachfrage ihre Sorgen mitgeteilt:

2. sollen wir Stellung dazu nehmen in der Sache: Schwangerschaftsunterbrechung. Sie wissen, dass wir Schwestern im Frauenspital nicht, auch nie im Mutterhaus reden durften über unsre Arbeit. Ich fragte mich oft, ist es recht, was in unserm Hause geschieht? Im Vertrauen aber zu unsern Vorgesetzten im Mutterhaus, die ja gewiss orientiert waren über unsere Arbeit, im Gedanken daran, dass ja in unserm Krankenhaus in Riehen, dieser Eingriff auch öfters vorgenommen wird; im Vertrauen auf unsre guten Ärzte, die ja für das Wohl der Menschheit nur das Beste wollen, habe ich mich in dieser Sache der Verantwortung frei gefühlt. In letzter Zeit habe ich mich oft empört, dass diese Operation so oft vorgenommen wird. $^{28}$

Die Zunahme der Abtreibungen wurde also deutlich empfunden. Obwohl sie sich als Krankenschwester der Verantwortung gegenüber den ärztlichen Verordnungen enthoben fühlte, befand sie sich in einem ethischen Dilemma: Ihre religiöse Überzeugung gebot ihr, ungeborenes Leben zu schützen, ihr pflegerisches Gewissen bedeutete ihr, jede Patientin ohne Vorbehalt zu betreuen und ihren Bedürfnissen auch in schwierigen Momenten gerecht zu werden. Gleichzeitig war sie als pflegende Schwester den Ärzten unterstellt und zur loyalen Ausführung der Anordnungen verpflichtet.

Diakonisse C. C. war nicht die einzige, die an dieser ethisch schwierigen Situation litt, die durch das ihr auferlegte Schweigen verschärft wurde. Pfarrer Hoch als Vertrauensperson der Diakonissen wusste davon, er brachte dies in seinem Schreiben an den Präsidenten der Aufsichtskommission, A. Christ-de Neufville, vor, als er Ende 1945 um die erneute Revision des Vertrags zwischen Frauenspital und Diakonissenanstalt ersuchte. Es sei vorgesehen, dass im Operationssaal freies Pflegepersonal einziehe, schrieb er, weil sich die Diakonissen «mehr und mehr in ihrem Gewissen belastet fühl-

28 Archiv: Diakonisse C. C. an Pfarrer Hoch, 10.8.1945. 
ten durch die zunehmende Zahl von Schwangerschaftsabbrüchen». Und er fuhr mit deutlichen Worten fort:

Wir instruieren zwar unsre Diakonissen in dem Sinne, dass Diagnose und Therapie, also auch die Entscheidung über vorzunehmende Operationen in die Verantwortung des Arztes und nicht der Schwester fallen. Wenn wir aber bedenken, in welche Verbrechen Schwestern in Deutschland mit einbezogen worden sind, indem sie sich, gedeckt durch diesen Grundsatz, $z u$ den Tötungen sogenannten 〈lebensunwerten Lebens〉 anstellen liessen, so halten wir es für dringend nötig, unsre Diakonissen dahin zu erziehen, dass sie auch auf ihr eigenes Gewissen achten. Wenn sich ihr christlich und mütterlich empfindendes Gewissen gegen die überhandnehmenden Schwangerschaftsunterbrechungen auflehnt, und sie uns ersuchen, sie aus dieser Arbeit zu befreien, so wissen wir uns verpflichtet, dieser Bitte zu entsprechen. ${ }^{29}$

Und er ergänzte, dass auch die pflegenden und leitenden Schwestern Kritik äusserten und weitere Versetzungen gewünscht worden waren, da sie «die Verantwortung für diese Abteilung gerade auch im Blick auf die häufigen Unterbrechungen vor ihrem Gewissen nicht übernehmen $»^{30}$ könnten.

Pfarrer Hoch argumentierte hier auf dem Hintergrund der nationalsozialistischen Verbrechen und der Mitverantwortung der deutschen evangelischen Diakonie ${ }^{31}$ und bezog sich konkret auf die «Euthanasie»-Programme und die Beteiligung der Pflegenden ${ }^{32}$ an den Patiententötungen. Damit begründete er, warum er die Bedenken der Diakonissen ernst nahm und ihr Verantwortungsgefühl nicht negieren wollte ${ }^{33}$. Für ihn bestand die Aufgabe der Pflegenden nicht darin, unhinterfragt jede ärztliche Anweisung auszuführen, sondern auf Wissen und innerer Überzeugung abgestützt mitzutragen. Die implizite Gleichsetzung der nationalsozialistischen «Euthanasie»-Programme mit Abtreibungen aus medizinisch indizierten Gründen mit Einwilligung der Betroffenen verweist in dieser Frage auf eine fundamentalistisch anmutende Überzeugung, wie sie auch katholisch-konservative Kreise vertraten, die aber in Basel an gesellschaftlichem Einfluss verlor.

Die Lösung des Konflikts, in dem sich unterschiedliche Weltanschauungen manifestierten, bestand schliesslich in der Vertragskündigung des Diakonissenhauses. Im Verlauf des Jahres 1946 verliessen die Diakonissen die gynäkologische Abteilung des Basler Frauenspitals. An der Sitzung der Aufsichts-

29 Archiv: Pfr. Hoch an den Präsidenten der Aufsichtskommission des Frauenspitals, A. Christde Neufville, 28.12.1945 (Hervorhebungen von S. B.).

30 Archiv: Pfr. Hoch an den Präsidenten der Aufsichtskommission des Frauenspitals, A. Christde Neufville, 28.12.1945.

31 Die deutschen und österreichischen Diakonissenhäuser hatten sich 1934 als «Diakoniegemeinschaft» in die «Reichsfachschaft deutscher Schwestern» eingegliedert und «die Arbeitsfront des deutschen Volkes» unterstützt. Vgl. «Mitteilungen», in: Diakonissenbote aus Riehen, 1934, 209.

32 Vgl. Steppe 1996.

33 Pfarrer Hoch hatte bereits 1942 an der Konferenz der schweizerischen Diakonissenhäuser in Riehen vom 26./27.1.1942 «Zur Frage der Euthanasie und der Vernichtung unwerten Lebens» einen Vortrag gehalten. Vgl. Diakonissenbote aus Riehen, 1942, 257, 55. 
kommission im Oktober 1946 ging Prof. Koller kurz auf die Vorgeschichte ein, die zum Rückzug der Diakonissen geführt habe.

Das Argument betr. Schwangerschaftsunterbrechung sei eigentlich nur vorgeschoben worden, diese Frage sei heute gelöst. Aus der Unterredung, die Präs. Christ und er mit Herrn Dr. Iselin von der Diakonissenanstalt hatten, konnte der Schluss gezogen werden, dass Riehen nicht mehr über genügend Schwestern verfügt, um die grössern Spitäler damit dotieren zu können ${ }^{34}$.

\section{Nachwirkungen}

Dieser ethische Konflikt, in dem sich die Diakonissen bei der Pflege von Frauen nach einer Schwangerschaftsunterbrechung sahen, wurde erst Jahre danach thematisiert. Im Bericht, den jede Diakonisse beim 50-Jahr-Jubiläum ihrer Einsegnung verfassen musste, schrieb A. R., die von 1902 bis 1946 im Frauenspital gewesen war und ab 1927 die Gynäkologie geleitet hatte: «Dann führte mich der Weg ins Frauenspital. Was kann ich von dort erzählen, ich wurde ja zum Stillesein erzogen» ${ }^{35}$. Auch in ihrem Rückblick 1952 konnte Schwester A. R. ihre inneren Kämpfe nur andeuten. Über ihre Erfahrungen konnte oder wollte sie nichts Näheres erzählen. Es scheint, dass auch die Schwestern untereinander kaum über diese Thematik diskutierten, denn in den Protokollen der Schwesternkonferenzen, die seit 1926 regelmässig abgehalten wurden, findet sich kein Hinweis darauf ${ }^{36}$, das Thema der Schwangerschaftsabbrüche scheint auch unter den Schwestern ein Tabu gewesen zu sein.

Ihre Nachfolgerin E. R. indes ging 1967 anlässlich ihres Berichts zum 50-Jahr-Jubiläum auf ihr Dilemma ein:

Ungefähr zur selben Zeit trat die Annahme des neuen Gesetzes der Schwangerschaftsunterbrechung in Kraft [1942]. Das hat ganz besonders für mich eine schwere Belastung gebracht. Die Fälle, die vorher sehr einzeln, und von Herrn Prof. Labhardt wohl geprüft durchgeführt wurden, mehrten sich sehr. Jetzt entschied das Gesetz. Die Gutachten, die auch bei mir zirkulierten, wurden eine Gewissensfrage für mich. [...] Nach einer Aussprache bei Herrn Prof. Koller ging ich mit meinen Anliegen zu Herrn Pfr. Hoch. Er besprach sich darauf auf Rat von Prof. Koller mit dem Chef der psychiatrischen Klinik. Denn von dort kamen die für mich unverständlichen Gutachten. Sein Vorschlag war, man solle die Schwestern mehr wechseln, wenn sie dieser Dienst seelisch belaste. Das war natürlich keine Lösung. Als es sich im Mutterhaus bald wieder um die Kündigung einer Station handelte, bat ich, dies mit der unsrigen zu tun. [...] Was mir über das Schwere des Wegganges hinweg half, war, dass die Schwangerenfürsorge dadurch ganz anders ausgeführt und ausgebaut wurde ${ }^{37}$.

34 StABS, SD-REG1 0-1-3: Protokoll der Aufsichtskommission des Frauenspitals Basel, 2.10.1946.

35 Archiv: Dossier von Diakonisse A. R., 1952.

36 Mündliche Auskunft der Archivarin des Diakonissenhauses, 31.1.2007.

37 Archiv: Dossier von Diakonisse E. R., 1967. 
Diakonisse E. R. hatte als Vizeoberschwester von 1925 bis 1946 im Operationssaal der gynäkologischen Abteilung gearbeitet und beschrieb in ihrem Rückblick auf ihr Diakonissenleben die schwierigen Momente ihres Pflegedienstes. Auch ihr machte der Umstand, dass sie nicht reden durfte, offensichtlich zu schaffen. Sie erlebte nach der Einführung des eidgenössischen Strafgesetzbuches von 1942, das mit dem Paragraphen 120 den Abort aus medizinischen Gründen legalisierte, die darauf folgenden Auswirkungen konkret in ihrem Tätigkeitsfeld. Der gesellschaftliche Wandel war nun im «Gesetz» festgehalten, gegen das sie sich machtlos fühlte.

In ihrem Rückblick sprach sie die für sie «unverständlichen» psychiatrischen Gutachten an. Was ihr daran unverständlich blieb, präzisierte sie nicht. Nach Einführung des StGB stiegen auch die Gutachtenzahlen rapid an ${ }^{38}$. Innerhalb von drei Jahren verdoppelten sie sich, wobei die Empfehlung für einen Abort bei 30 bis 50 Prozent lag. Um die Nachfrage nach legalen Schwangerschaftsabbrüchen zu bewältigen, wurde auf Antrag des Direktors der psychiatrischen Institutionen die Zahl der für die Gutachtenerstellung berechtigten Psychiater und Psychiaterinnen 1943 erhöht.

Auch freien Berufskrankenschwestern fiel das Assistieren bei Abtreibungen nicht leicht, wie E. R.s Nachfolgerin Rosa Leuppi in ihrem Tagebuch über ihr gesamtes Berufsleben festhielt ${ }^{39}$. Sie stellte sich eingehend die Frage, wie sie als katholisch sozialisierte Krankenschwester damit umgehen sollte, bevor sie die Leitung der gynäkologischen Abteilung im Basler Frauenspital übernahm. Es sei allgemein bekannt gewesen, dass die Diakonissen auch deswegen gekündigt hätten und nicht nur aufgrund des Schwesternmangels, schrieb sie und ergänzte, dass auch der Spitalpfarrer sie vor den zahlreichen Schwangerschaftsabbrüchen gewarnt habe. Ein Gespräch mit ihrem ehemaligen Arbeitgeber Prof. Suter, der die Basler Privatklinik «Sonnenrain» leitete, zerstreute allerdings ihre Zweifel: «Zwar sei es für das Pflegepersonal keine erfreuliche Sache, doch läge die Verantwortung letztlich beim Arzt» ${ }^{40}$. Um ihre moralischen Bedenken bei den Abtreibungen zu vermindern, engagierte sie sich stark in der Beratungsstelle für werdende Mütter, die die Frauen so unterstützen sollte, dass sie das Kind austrugen ${ }^{41}$.

$38 \mathrm{Vgl}$. Im folgenden: Imboden/Ritter 2007.

39 Vgl. Spirig-Zünd 1998, $120 \mathrm{f}$.

40 Spirig-Zünd 1998, 123.

41 Vgl. StABS, GGG, PA 146 Q 25, Beratungsstelle für werdende Mütter: Jahresbericht 1948 und 1950 sowie Spirig-Zünd 1998, 132. 


\section{Schlussbemerkungen}

Aufgrund ihrer religiösen Überzeugung und ihres durch Gehorsam und Schweigen geprägten Schwesternideals gerieten die Diakonissen in ein ethisches Dilemma um die Zunahme der Abtreibungen. Hatte der Schutz des ungeborenen Lebens Vorrang vor der Gesundheit oder dem Willen der schwangeren Frau, das Kind nicht auszutragen? Ihre christliche Moral gebot ihnen, das ungeborene Leben zu schützen, ihre pflegerische Verpflichtung verlangte jedoch die vorbehaltlose Betreuung der Patientin vor, während und nach einem medizinischen Eingriff. Als Ausweg bot sich ihnen die «Schwesternnot», der akute Pflegepersonalmangel, der dazu führte, dass sie ins Diakonissenhaus zurückgerufen und in anderen Spitälern, wo Personalmangel herrschte, eingesetzt wurden. So fanden die Diakonissen auf individueller Ebene eine Lösung ihres ethischen Dilemmas.

Auf der gesellschaftlichen Ebene werden in diesem Konflikt, der sich im Basler Frauenspital um die Zunahme der Schwangerschaftsabbrüche geräuschvoll abspielte, unterschiedliche Auffassungen über Geburtenregelung und freiwillige Geburtenbeschränkung sichtbar. Während sich in Basel schon früh ein gesellschaftlicher Wandel hin zu grösserer Entscheidungsfreiheit für Frauen abzeichnete, blieb auf der eidgenössischen Ebene das Konfliktpotential um die Frage der Abtreibung bestehen und verhinderte bis ins Jahr 2002, dass Frauen aufgrund einer freien und selbstverantwortlichen Entscheidung eine Schwangerschaft in den ersten 12 Wochen abbrechen konnten ${ }^{42}$. Nachdem das Schweizer Stimmvolk die Änderung des Artikels 120 des Strafgesetzbuches (die Fristenlösung) am 2.6.2002 angenommen hat, ist diese Frage in der Schweiz geklärt.

\section{Bibliographie}

Berger, J./K. Stäuble, «Theo Hermann Koller», in: Koller, Th./H. Stamm/K. Stäuble (Hrsg.), 100 Jahre Geburtshilfe und Gynäkologie in Basel, 1868-1968 (Basel 1970) 267-272

Braunschweig, Sabine, Aufbruch und Pioniergeist. Chronik der Schule für Spitalberufe, Hrsg. Berufsschule für Pflege Kanton Basel-Landschaft (Liestal 1998)

Dalonni, Marcelle, «Arzt und Schwester», Diakonissenbote aus Riehen (1943) 264,109-111

Freudweiler, Emmy/Emmy Oser, Die Arbeitsverhältnisse der Krankenpflegerinnen in der Schweiz, Hrsg. Union für Frauenbestrebungen (Zürich 1913)

Heller, Geneviève/Gilles Jeanmonod/Jacques Gasser, Rejetées, rebelles, mal adaptées. Débats sur l'eugenisme. Pratiques de la stérilisation non volontaire en Suisse romande au $X X^{e}$ siècle (Genf 2002)

42 Bei der Gesetzesberatung 2001 wurde im Parlament auch die umstrittene Frage eines psychiatrischen Gutachtens für einen Schwangerschaftsabbruch abgelehnt. 
Imboden, Gabriela/Hans Jakob Ritter et al., «Abtreibung und Sterilisation - Psychiatrie und Geburtenkontrolle im Kanton Basel-Stadt zwischen 1920 und 1960», in: Mottier,V./L.v. Mandach (Hrsg.), Pflege, Stigmatisierung und Eugenik. Integration und Ausschluss in Medizin, Psychiatrie und Sozialhilfe (Zürich 2007) 38-50

Joris, Elisabeth/Heidi Witzig (Hrsg.), Frauengeschichte(n). Dokumente aus zwei Jahrhunderten zur Situation der Frauen in der Schweiz (Zürich 1986)

Kellerhals, Sr. Doris/Lukrezia Seiler/Christine Stuber (Hrsg.), Zeichen der Hoffnung. Schwesterngemeinschaft unterwegs (Basel 2002)

Koller, Theo/Heinrich Willi, Die Gesundherhaltung von Mutter und Kind (Zürich 1939) (291971)

Labhardt, Alfred, «Straflose Abtreibung im Kanton Basel-Stadt», Correspondenzblatt für Schweizer Aerzte (1919) 1078-1084

Mall-Haefeli, Marianne, «Die sozialmedizinische Abteilung am Frauenspital», in: Koller, Th./H. Stamm/K. Stäuble (Hrsg.), 100 Jahre Geburtshilfe und Gynäkologie in Basel, 18681968 (Basel 1970) 412-420

Spirig-Zünd, Jolanda, Kaffee mit Muttermilch. Erinnerungen der Krankenschwester Rosa Leuppi (Zürich 1998)

Steppe, Hilde (Hrsg.), Krankenpflege im Nationalsozialismus (Frankfurt a.M. 1996)

Sticker, Anna, Die Entstehung der neuzeitlichen Krankenpflege (Stuttgart 1960) 